\title{
High Order Bragg Gratings for Short Wavelengths Operation
}

\author{
Jing He*, Yun Wang, Martynas Beresna, Gilberto Brambilla \\ Optoelectronics Research Centre, University of Southampton, Southampton, SO17 1BJ, UK \\ *Author e-mail address: J.He@ soton.ac.uk
}

\begin{abstract}
High order gratings using phase mask technique are investigated theoretically and experimentally. The results shows that even a small amount of the zerothorder component in the diffraction distribution would have substantial effects on high order gratings. It provides a useful basis for producing different gratings towards short wavelength application using one single phase mask.
\end{abstract}

Keywords: Fiber Bragg Grating, Second-order grating, Interference, Index modulation

\section{INTRODUCTION}

Fiber Bragg gratings (FBG) are attractive optical devices with a wide range of applications in laser systems [1], optical telecommunications [2], and sensors [3]. The phase mask technique has been widely used in manufacturing of fiber gratings [4] and represents the most common technique for mass production, as it greatly simplifies the manufacture process and produces gratings with high performance. However, once the pitch of a phase mask is chosen, it is difficult to tune the Bragg wavelength over more than $100 \mathrm{~nm}$. Additionally, due to the limitation of the phase mask manufacturing technology, small pitch for short wavelength operation, especially in the UV band, is hard to achieve. In 1993, B. Malo et al. observed excimer-pulse-written high order gratings by use of a zeroth-order nulled phase mask [5]. Nevertheless, the presence of the 0th order diffraction beam, which interferes with \pm 1 st orders and causes the formation of a periodic Talbot interference pattern [6]. It affects the intensity distribution behind the phase mask, changing the FBG properties.

In this paper, high order gratings are optimized for short wavelength operation through both numerical investigation and experiments demonstration. A growth modeling of the photo-imprinting process of FBGs combining with 0th order diffraction effects is proposed. The model is based on the exponential dependence of the photo-induced refractive index changing with exposure time and incident optical power. The saturation effect of the induced index is also considered. The experiment is carried out in Ge-doped fiber under the exposure of UV femtosecond radiation, which is associated to multiphoton absorption process in the mechanism for photo- induced index change [7], ensuring the effectivity and stability of high order gratings in short wavelength range.

\section{THEORETICAL ANALYSIS}

We are assuming, that a quasi-monochromatic plane wave polarized parallel to the grating grooves is incident normally on a phase mask of period $\Lambda_{\mathrm{PM}}$. The emergent field distribution behind the phase mask is composed of a set of diffracted plane waves $[6,8]$ propagating at angles $\psi_{n}$, satisfying

$$
\sin \psi_{n}=\frac{n \lambda}{\Lambda_{P M}}, n=0, \pm 1, \pm 2, \ldots, \pm m
$$

where $\lambda$ is the imposed wavelength in the medium and $\mathrm{m}$ is the largest integer for which $\psi_{n}$ retains a real value. The $\mathrm{z}$ direction denotes the grooves array direction and the $\mathrm{x}$ direction the laser beam incident direction on the phase mask. The contributions from each diffracted component, ignoring vector effects, can be superposed to generate the field, $\mathrm{E}(\mathrm{x}, \mathrm{z})$ produced by the interference of diffracted beams [9]

$$
E(x, z)=\sum_{m} C_{m} \mathrm{e}^{i k_{m} x} \mathrm{e}^{i m \mathrm{Gz}}
$$

where $C_{m}$ is the amplitude of the electric field of the mth order diffraction, $k_{m}=\left(k^{2}-m^{2} G^{2}\right)^{1 / 2}$ is the $\mathrm{x}$ component of wave vector $\mathrm{k}, G=2 \pi / \Lambda_{P M}$ is the reciprocal vector of the phase mask, wave vector $k=$ $2 \pi / \lambda$.

The index change profile of FBG can be regarded as a function of $\mathrm{z}$ and $\mathrm{t}$, and it is periodic in $\mathrm{z}$ with a constant period, also influenced by the exposure time $t$ and intensity distribution imposed by $\mathrm{E}(\mathrm{x}, \mathrm{z})$. Here, for the sake of simplicity, the diffraction orders higher than \pm 1 th are ignored. Due to the unavoidable 0th-order diffraction, gratings will be effected by the interference between \pm 1 th order and 0th order. Considering the exponential dependence of the photo-induced refractive index changing and the saturation effect $[10,11]$, the model of FBG index variation can be described by

$$
\begin{gathered}
n(z, t)=n_{c o}+\delta n(z, t) \\
\delta n(z, t)=\delta n_{0}(1- \\
\left.e^{k_{1} \cdot t\left(I_{1} \cdot \cos ^{2}\left(\frac{2 \pi z}{\Lambda_{P M}}\right)+I_{0}+2 \sqrt{I_{0} I_{1}} \cos \left(\frac{2 \pi z}{\Lambda_{P M}}\right) \cos \left(\frac{2 \pi x}{\lambda}\left(1-\sqrt{1-\left(\frac{\lambda}{\Lambda_{P M}}\right)^{2}}\right)\right)\right)}\right)
\end{gathered}
$$


Where $n_{c o}$ is the refractive index inside the fiber core, $\delta n(z, t)$ represents the photo-induced refractive index change, $\delta n_{0}$ is the saturation level, i.e. the maximum variation of index change, $k_{1}$ is an adjustable parameter proportional to the intensity of the UV beam, $I_{1}$ represents the symmetrical intensity of \pm 1 th order diffraction, and $I_{0}$ represents the intensity of the 0th order diffraction through phase mask.

The index variation function $\delta n(z, t)$ can be expanded into Fourier series,

$$
\begin{gathered}
\delta n(z)=\delta n_{0}\left(1+\delta n_{1} \cos \left(\frac{2 \pi z}{\Lambda}+\beta_{1}\right)\right. \\
+\delta n_{2} \cos \left(\frac{4 \pi z}{\Lambda}+\beta_{2}\right) \cdots \\
\left.+\delta n_{N} \cos \left(\frac{2 \pi z}{\Lambda} \cdot N+\beta_{N}\right)\right) \\
0 \leq z \leq L \\
\\
\delta n(z)=0, z>L
\end{gathered}
$$

Where the $\delta n_{N}$ values determine the peak reflectivity of $\mathrm{N}$-th order grating, $\mathrm{L}$ represents the total length of the grating.

To simulate a realistic case, a phase mask of period $\Lambda_{\mathrm{PM}}=1074 \mathrm{~nm}$ designed for $\lambda_{B}=1550 \mathrm{~nm}$, and a standard telecom fiber with radius of $x=62.5 \mu \mathrm{m}$ are chosen. To simplify the simulation, we define $\mathrm{T}=k_{1} t I_{1}=0.5,1,5,20$, and $e=\sqrt{\frac{I_{0}}{I_{1}}}=0.1$. It can be observed from Fig. 1 (a) that instead of having a period of $\Lambda=\Lambda_{\mathrm{PM}} / 2$ in the case of 0 th-order nulled phase mask [12], the index change fringes are spaced by period of $\Lambda=\Lambda_{\mathrm{PM}}$. With the increase of the T, i.e. extending the exposure time or incident radiation power, the fringe visibility is decreasing progressively.

This effects is further accentuated when e increases to 0.3 as shown in Fig. 1 (b). The result proves that even with small efficiency in the diffraction field, the 0th order component causes a huge effect on the FBG resonant wavelength.

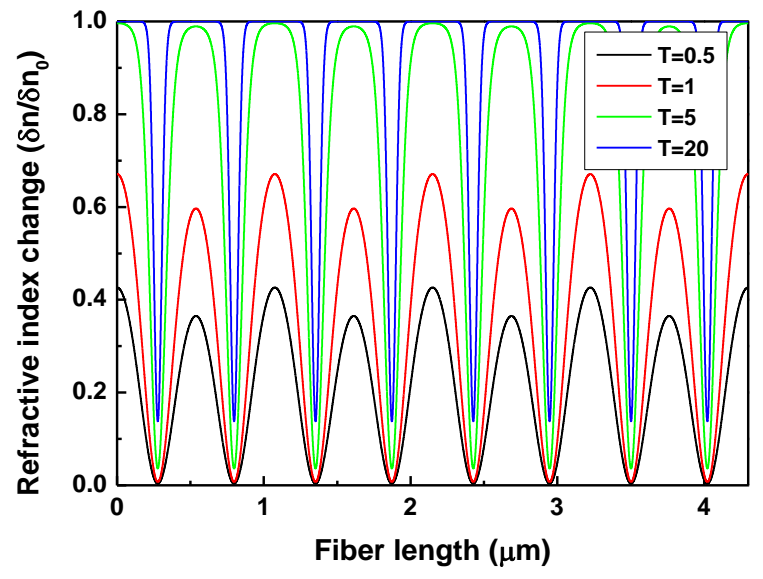

(a)

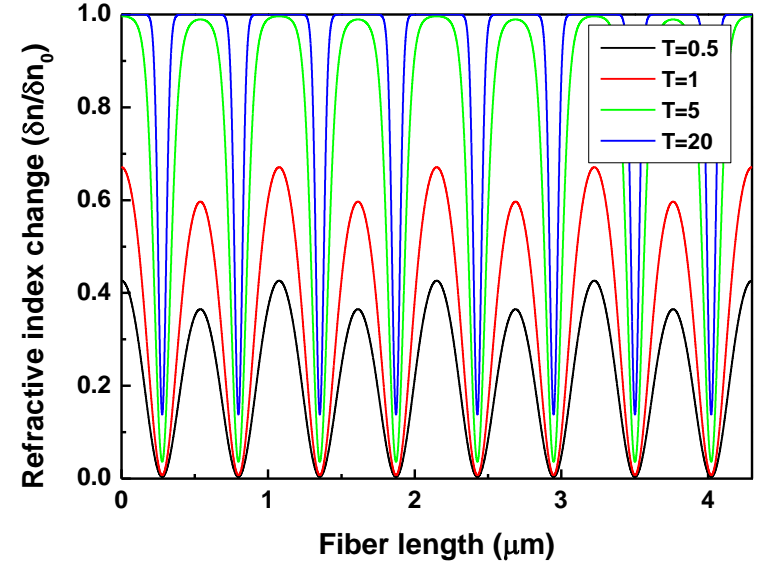

(b)

Fig. 1 Refractive index change along the FBG (a) $\mathrm{e}=0.1$; (b) $\mathrm{e}=0.3$

Fig. 2 gives the refractive index change of each higher order gratings versus with defined exposure time $\mathrm{T}$. There are changes of high order gratings both in the resonant wavelengths and intensity of reaching the maximum point.

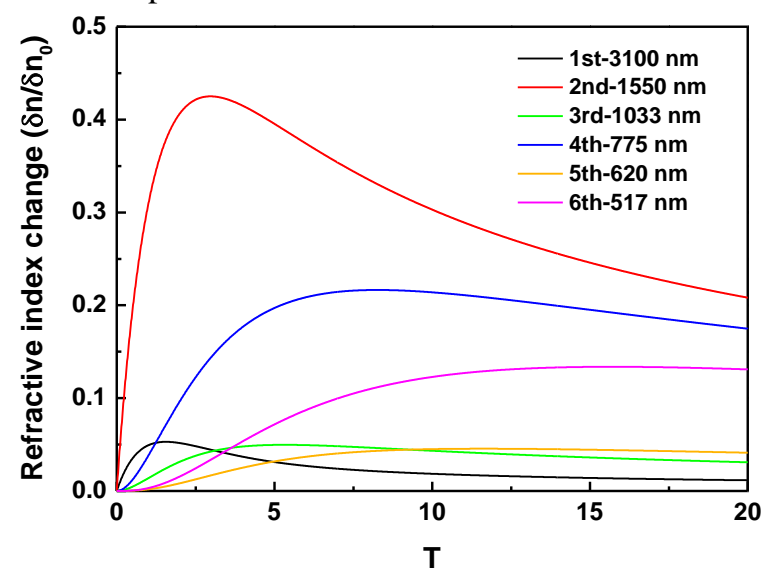

(a)

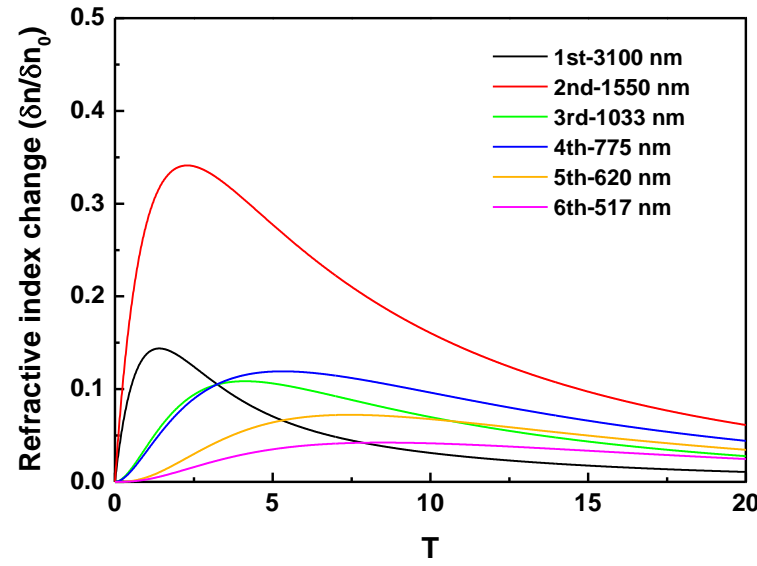

(b)

Fig. 2 Refractive index change with the increasing of T (a) $\mathrm{e}=0.1$; (b) $\mathrm{e}=0.3$

The appearance of a peak at the Bragg wavelength $\lambda_{B}=3100 \mathrm{~nm}$ can be attributed to the 0th order interference influence on fringe period of $\Lambda_{\mathrm{PM}}$. This interference also explains the emergence of peaks at 
$1033 \mathrm{~nm}$ and $620 \mathrm{~nm}$, which are not commonly observed in higher order gratings. When e increase from 0.1 to 0.3 , the even-th order components $(1550 \mathrm{~nm}, 775 \mathrm{~nm}$, and $517 \mathrm{~nm}$ ) experience a significant drop of the index change, corresponding to a decrease of reflectivity, making them a weaker grating, while the odd-th order enjoys a growth. That means developing 0th order diffraction can be used for strengthen the odd-th order gratings, and to reduce the even-th order ones.

\section{EXPERIMENTAL RESULTS}

The method for producing the Bragg gratings is similar to that described by Stephen A. et al. [13]. The fourthharmonic radiation of a femtosecond laser emitting 220 fs pulses at $258 \mathrm{~nm}$ is used for the inscription of FBGs. The measured beam diameter is $0.3 \mathrm{~cm}$ (FWHM), the repetition rate is set to be $1 \mathrm{kHz}$, and the pulse energy is up to $80 \mu \mathrm{J}$. In the fabrication process we used the phase mask from Ibsen Photonics with a period of $\Lambda=1.074$ $\mu \mathrm{m}$ resulting in primary Bragg peak at $\lambda_{\mathrm{B}}=1556 \mathrm{~nm}$ in a photosensitive fiber (PS750).

Fig. 3 shows the evolution of the reflection spectrum of the second order grating around $783 \mathrm{~nm}$ during the inscription process. The insert of Fig. 3 gives the dependence of Bragg wavelength and strength with the exposure time. When exposure time increases from 200 $\mathrm{s}$ to $1400 \mathrm{~s}$ on a total inscribed fiber length of $1 \mathrm{~cm}$, the strength of the second order grating firstly presents an exponential increase, and then a fast degradation after reaching the saturation point. In the meantime, the center wavelength of the grating moves towards the longer wavelengths through all the process, which is induced by the change of the average mode effective index [12, 13]. These experiments results perfectly verify the theoretical analysis.

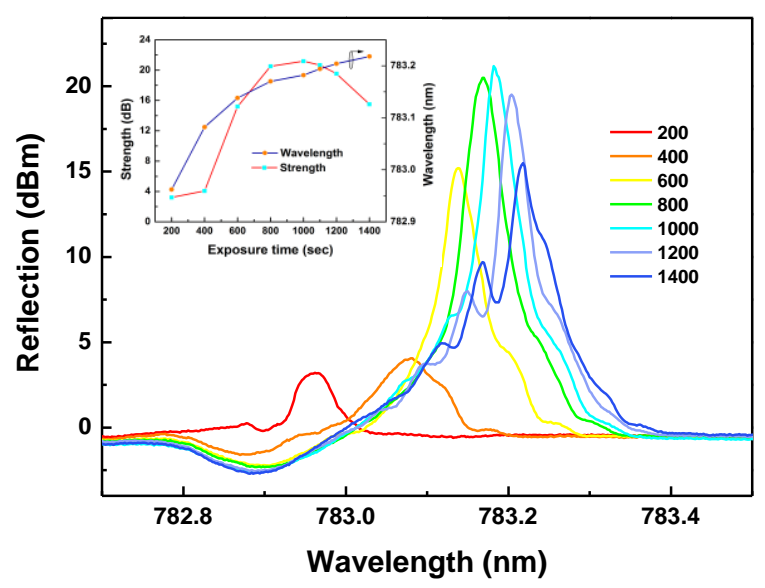

Fig. 3 Evolution of reflection spectra at $783 \mathrm{~nm}$ during inscription process

\section{CONCLUSION}

The analysis of high order gratings using phase mask technique is reported through both theoretically calculation and experiments demonstration. Modeling of the photo-imprinting process of FBGs based on the exponential dependence is studied. 0th-order diffraction effects and the saturation effect of the induced index are also considered. The results shows that even a small contributions of the 0th-order component would produce significant variations on high order gratings. The experimentally we demonstrated that reflectivity of second order can reach $20 \mathrm{dBm}$. The model provides valuable guidance to understand the grating formation and to produce higher order gratings for short wavelength application on one single phase mask.

\section{ACKNOWLEDGMENTS}

The authors gratefully acknowledge funding from EPSRC through the research grant EP/L01243X/1

\section{REFERENCES}

[1] G. A. Ball and W. W. Morey, "Compression-tuned singlefrequency Bragg grating fiber laser," Opt. Lett., vol. 19, pp. 1979-1981, 1994.

[2] A. Othonos, K. Kalli, and G. E. Kohnke, "Fiber Bragg gratings: Fundamentals and applications in telecommunications and sensing," Physics Today, vol. 53(5), pp. 61, 2000.

[3] A.D. Kersey, M.A. Davis, H.J. Patrick, et al., "Fiber grating sensors," J. Lightwave. Technol., vol. 15, pp. 1442-1463, 1997.

[4] K. O. Hill, B. Malo, and F. Bilodeau, et al., "Bragg gratings fabricated in monomode photosensitive optical fiber by UV exposure through a phase mask," Appl. Phys. Lett., vol. 62, pp. 1035-1037, 1993.

[5] B. Malo, D. C. Johnson, and F. Bilodeau, et al. "Singleexcimer-pulse writing of fiber gratings by use of a zeroorder nulled phase mask: grating spectral response and visualization of index perturbations," Opt. Lett., vol. 18, pp. 1277-1279, 1993.

[6] Z. Xiong, G. D. Peng, B. Wu, and P. L. Chu, "Effects of the zeroth-order diffraction of a phase mask on Bragg gratings," J. Lightwave Technol., vol. 17, pp. 2361-2365, 1999.

[7] S. J. Mihailov, C. W. Smelser, P. Lu, et al., "Fiber Bragg gratings made with a phase mask and 800-nm femtosecond radiation," Opt. Lett., vol. 28, pp. 995-997, 2003.

[8] P. E. Dyer, R. J. Farley, and R. Giedl, "Analysis of grating formation with excimer laser irradiated phase masks," Opt. Commun. vol. 115, pp. 327-334, 1995.

[9] C. Kittel, Introduction to Solid State Physics, 6th ed. John Wiley \& Sons, New York, 1986, Chap. 2.

[10] D. Z. Anderson, V. Mizrahi, T. Erdogan, and A.E. White, "Production of in-fibre gratings using a diffractive optical element," Electron. Lett., vol. 29, pp. 566-568, 1993.

[11] W. X. Xie, M. Douay, P. Bernage, et al., "Second order diffraction efficiency of Bragg gratings written within germanosilicate fibres," Opt. Commun. vol. 101, pp. 8591, 1993.

[12] R. Kashyap, Fiber Bragg Gratings, Academic Press, San Diego, CA, 1999, Chap. 8.

[13] S. A. Slattery, D. N. Nikogosyan, and G. Brambilla, "Fiber Bragg grating inscription by high-intensity femtosecond UV laser light: comparison with other existing methods of fabrication," J. Opt. Soc. Am. B., vol. 22, pp. 354-361, 2005. 